\title{
EXPERIENCIA EN CAPACITACIÓN Y TRANSFERENCIA DE HERRAMIENTAS DIDÁCTICAS SOBRE EL USO RACIONAL DE LA ENERGÍA Y ENERGÍAS RENOVABLES
}

Eje 3: Extensión universitaria y transferencia tecnológica

Cisterna M. Susana ${ }^{1}$

Ledesma Sara Lia²

Martínez Cecilia F. ${ }^{3}$

Nota Viviana M. ${ }^{4}$

Quiñones Graciela I. ${ }^{5}$

Márquez Vega S. Gabriela 6

Llabra Cristina ${ }^{7}$

\author{
Colaboradores: M Mostajo, M Ramos, S Atencia, C Villa
}

1,2,3,4,5,6,7 Centro de Estudios Energía, Habitabilidad y Arquitectura Sustentable. Instituto de Acondicionamiento Ambiental, Facultad de Arquitectura y Urbanismo, Universidad Nacional de Tucumán, San Miguel de Tucumán, Tucumán, Argentina, sledesma@herrera.unt.edu.ar, mscisterna@hotmail.com, cfernandamartinez@gmail.com

\section{RESUMEN}

Se presentan las actividades desarrolladas en el marco del Proyecto de Extensión "Capacitación y transferencia sobre mejoramiento de condiciones ambientales, uso racional de energía y energías renovables", Ilevado a cabo por docentes de la cátedra de Acondicionamiento Ambiental I de la Facultad de Arquitectura y Urbanismo de la Universidad Nacional de Tucumán, con el fin de impulsar la incorporación de la temática energético-ambiental a través de contenidos y actividades en escuelas públicas de Tucumán. Las actividades planteadas buscan promover el uso racional de la energía y la aplicación de energías renovables para ahorrar energía en la escuela y en la propia casa. Se trabajó en forma articulada con la Secretaria de Medio Ambiente de la Provincia, quien a través de su "Equipo Provincial de Educación Ambiental" brindó la vinculación y coordinación con las escuelas de la Provincia. En la presentación se muestran los resultados de una experiencia piloto desarrollada con participación de directivos, docentes y alumnos de $4^{\circ}$ y $5^{\circ}$ grado de la escuela primaria Capitán de los Andes de nuestra provincia. Las actividades incluyeron el diseño y prueba de herramientas didácticas y transferencia del material para la implementación de la experiencia por parte de los docentes de la escuela. La experiencia piloto abarcó el planteo de contenidos y actividades en relación a las necesidades detectadas en la 
curricula actual, dictado de clases con apoyo audiovisual y talleres demostrativos de sistemas con aplicación de energía solar. Los principales contenidos abordados en las clases incluyen los tipos y formas de aplicación de la energía, su relación con el cambio climático. Sistemas y aplicación de energías renovables, la energía en los edificios, uso racional, posible ahorro y mejoras en las condiciones de habitabilidad. Sistemas con aplicación de energía renovable, con utilización de diferentes materiales y accesorios. También se incluyen talleres con modelos demostrativos de dos sistemas con energía solar: Horno Solar y Colector de Agua, con preparación de alimentos y de agua caliente sanitaria, factibles de ser construidos y utilizados en la escuela y en la propia casa. Conclusiones: Las actividades desarrolladas permitieron conocer las necesidades, transferir conocimiento y desarrollar pensamiento crítico en el contexto de la educación escolar primaria de Tucumán, en pro de una conciencia ambiental más eficiente que considere y evalúe en profundidad, sobre bases científicas, la incorporación de energías renovables y formas de uso racional de la energía en los ámbitos donde desarrolla diariamente sus actividades.

\section{PALABRAS CLAVES: ESTRATEGIA PEDAGÓGICA - ENERGÍAS RENOVABLES - TUCUMÁN}

\section{INTRODUCCIÓN}

La humanidad se encuentra ante el desafío de tener que cambiar sus paradigmas culturales para alcanzar un futuro sustentable, basado en el respeto por el medio ambiente y la responsabilidad en el uso de sus recursos. La educación es uno de los motores que puede ayudar en la producción de ese cambio. La complejidad de la problemática energético-ambiental hace imposible encontrar una respuesta aislada, específica e independiente para las partes implicadas. Es fundamental buscar soluciones integrales que consideren las interacciones de los sistemas (naturales y artificiales) entre sí y con las estructuras sociales FOLLARI, R. (1999). La educación, en sus distintos niveles, ofrece la posibilidad de abordar la compleja crisis socio-ambiental. Desde hace algunos años, importantes organismos como la UNESCO a través del PNUMA (Programa Internacional de Educación Ambiental) ponen énfasis en el desarrollo de las naciones a partir de la preservación de los recursos naturales y culturales, basado en principios de la educación ambiental, a través de enfoques interdisciplinarios. La educación como proceso permite adquirir conocimientos, desarrollar habilidades y actitudes en relación al ambiente; se plantea estimular la curiosidad, fomentar la toma de conciencia y orientar a las personas hacia un interés instruido que promoverá acciones positivas ROQUE MOLINA, M. (2003).

Si bien la educación es un proceso que dura toda la vida, durante la escolaridad primaria, los niños, instintivamente, tienen una visión holística del ambiente y es posible crear conciencia ambiental, actitudes, valores y compromiso en relación al uso racional de los recursos TORRES (2009); para desarrollar acciones y responsabilidades éticas para el desarrollo de su vida futura. Se asume también, el vínculo con la ética y los valores, al considerar a la Educación Ambiental un campo íntimamente vinculado con la formación cívica y ciudadana. E. GONZÁLEZ, L. FIGUEROA (2009).

A partir de la revolución industrial, el uso del carbón, primeramente, luego el petróleo y gas, ha crecido en forma permanente, por un lado debido al crecimiento de la población, y por otro, por el aumento del consumo de energía per cápita, consecuencia de un modelo económico que suele identificarse con bienestar. A pesar de las sucesivas crisis no se ha logrado armonizar el desarrollo económico, el mantenimiento de condiciones ambientales adecuadas y la presencia de 
una mayor equidad social. Una de las consecuencias de este elevado consumo es el calentamiento global del planeta debido en gran medida a la excesiva acumulación de los gases de efecto invernadero $\mathrm{GEI}$ - dióxido de carbono ( $\mathrm{CO} 2)$, metano $(\mathrm{CH} 4)$ - en la atmósfera. Se estima que el $65 \%$ de las emisiones de GEI, son consecuencia del uso de combustibles fósiles, de seguir así el consumo y la emisión, en un siglo más se alcanzaría un aumento de $2^{\circ} \mathrm{C}$ a $5^{\circ} \mathrm{C}$. Por lo tanto resulta necesario e imperioso disminuir las mencionadas emisiones.

En relación al hecho de que el consumo de energía guarda relación con el diseño, con las tipologías constructivas de los edificios y, fundamentalmente, con los hábitos de los usuarios, esta problemática adquiere una significativa complejidad, la cual es abordada en el proyecto. En función de lograr una mayor eficiencia energética en edificios, teniendo en cuenta la gran incidencia que en el consumo tienen la forma de uso y las costumbres de los usuarios, se plantea concientizar a los mismo sobre la importancia de la problemática y se propone su capacitación para reducir los consumos energéticos y mejorar las condiciones de habitabilidad y confort en las viviendas. I. BLASCO LUCAS (2006)

\section{DESARROLLO}

El proyecto fue desarrollado y llevado a cabo por docentes de la cátedra de Acondicionamiento Ambiental I de la Facultad de Arquitectura y Urbanismo de la Universidad Nacional de Tucumán. Surgió de la premisa de considerar a la educación como un proceso que permite a la sociedad desarrollar plenamente su capacidad crítica para alcanzar un desarrollo sostenible. Plantea mejorar las condiciones de habitabilidad y alcanzar un ahorro energético con una mejor calidad de vida en la escuela y en la casa, a partir de incorporar estrategias de calefacción y refrigeración pasiva, racionalizar el uso de la energía e incorporar energías renovables. Focalizados en este objetivo, considerando que el 30 \% del consumo de la energía proviene del área de la edificación donde el sector residencial representa el segundo mayor consumidor en el balance energético nacional (BEN, 2014) se ha desarrollado el proyecto de extensión con el objetivo de Incorporar la temática energético-ambiental en escuelas públicas de Tucumán.

El proyecto incluyó dos etapas, una basada en una experiencia que se realiza en la escuela, con la participación activa de la comunidad educativa y otra etapa que consiste en transferir el material necesario para que los docentes de la escuela, en el futuro, lleven adelante el dictado de clases y talleres sobre temática energético-ambiental. La experiencia piloto tiene como objetivo presentar, a través de clases y talleres, los contenidos, estrategias y técnicas, en interacción con los directivos, docentes y alumnos de $4^{\circ}$ y $5^{\circ}$ grado. Una vez concluida esta etapa se realizó la transferencia de material didáctico para que los docentes puedan replicar en el futuro el dictado en su escuela. La idea se basa en trabajar en forma coordinada con el Equipo Provincial de Educación Ambiental, para llevar a cabo nuevas experiencias y transferir material didáctico a otros establecimientos escolares de la Provincia de Tucumán.

El proyecto piloto incluyó clases con apoyo audiovisual, talleres demostrativos de sistemas con energía solar y de reflexión con expresión plástica y musical. Como una estrategia importante, se planteó la interacción con el personal directivo y docentes de la escuela, lo cual permitió conocer los temas y contenidos incluidos actualmente en la currícula, y también las necesidades e inquietudes de la comunidad. De esta manera, se buscó que el material transferido se adapte en gran medida a las características socio-culturales del colectivo al cual va dirigido, propiciando la aplicación de contenidos, estrategias y técnicas adecuadas para un aprendizaje eficiente. 


\subsection{DESCRIPCIÓN DE LA EXPERIENCIA. Articulación con la Secretaria de Medio Ambiente de la Provincia}

Se trabajó en forma articulada con la Secretaria de Medio Ambiente de la Provincia, quien a través de su "Equipo Provincial de Educación Ambiental" dirigido por la Dra. Florencia Sayago (Fig.1 y 2), brindó la vinculación y coordinación con escuelas de la Provincia. A través de la base de datos y de relevamientos realizados por la Secretaría se pudo conocer las escuelas que mostraban interés en la temática medio ambiental o que habían desarrollado proyectos y trabajos en temas relacionados con la misma. Entre los posibles establecimientos se eligió la escuela Capitán de Los Andes, ubicada en el barrio San Martín, al sudoeste de la Provincia de Tucumán. Se valoró para la selección el interés sobre la temática demostrado en los trabajos realizados por esta comunidad educativa en los últimos años, con motivo de participar en la Feria de Ciencia, a nivel provincial y nacional.
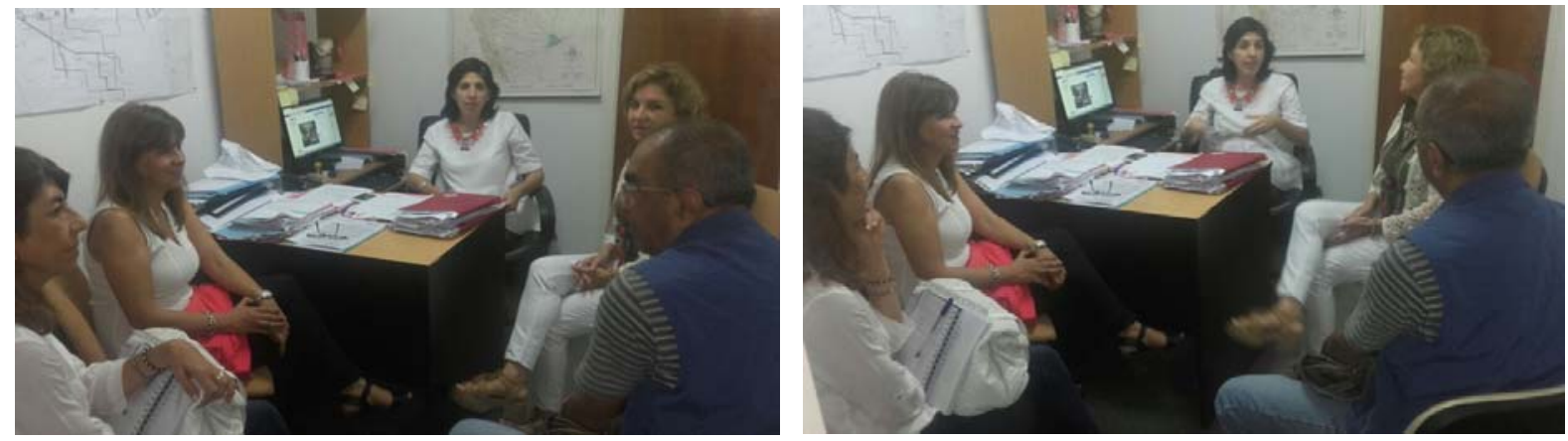

Fig. 1 y 2. Reunión con la directora de Medio Ambiente, Secretaría de Medio Ambiente de la Provincia de Tucumán.

La experiencia piloto se llevó a cabo con participación de directivos, docentes y alumnos de $4^{\circ}$ y $5^{\circ}$ grado. En forma conjunta, con la Directora del establecimiento, a través de entrevistas dirigidas a los docentes de grado y de materias especiales -Tecnología, Plástica y Música- se acordaron los contenidos a abordar en relación a los conocimientos sobre la temática energético-ambiental con que ya contaban los alumnos (Fig. 3, 4 y 5). Las entrevistas también sirvieron para conocer la situacion socio-económica del colectivo de alumnos, conocimiento que se aplicó en el enfoque de algunos contenidos, principalmente los relacionados con las condiciones de habitabilidad en los edificios.

En sucesivas reuniones se convinieron las fechas de cuatro clases con material audio-visual, una demostración con Horno Solar y con Colector solar de agua, y como actividad final de integración de contenidos, un taller de expresión plástica y musical para elaboración de trabajos sobre los temas abordados en las clases. 


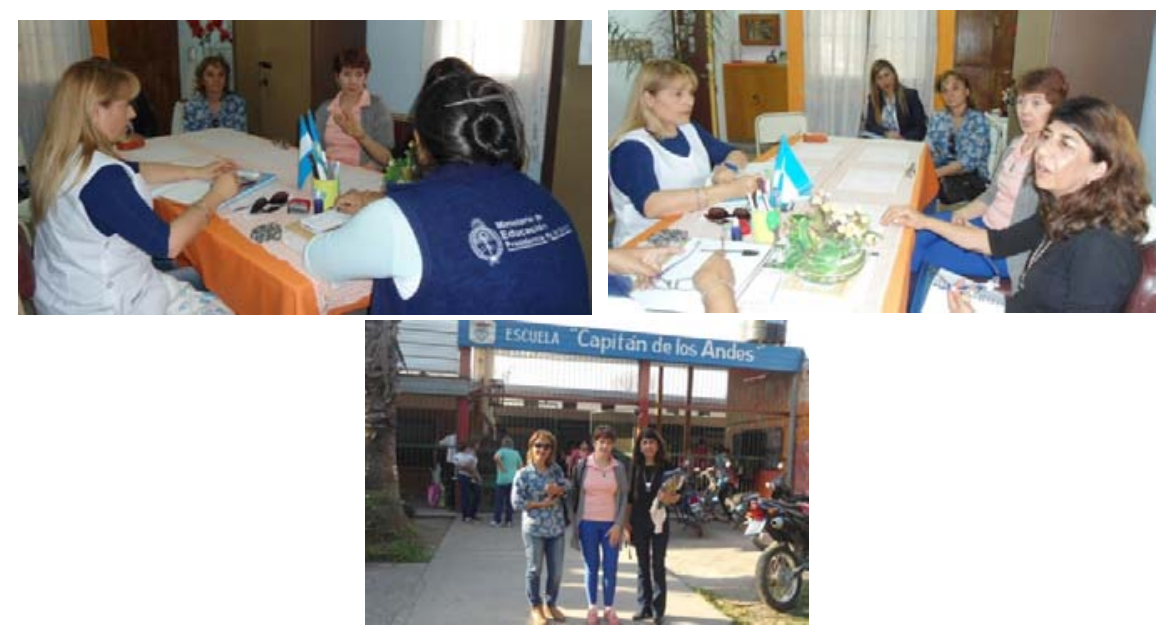

Fig. 3, 4 y 5. Reunión del equipo de trabajo con la Directora y docentes de la Escuela Capitán de los Andes

\subsection{OBJETIVOS ESPECÍFICOS Y ACTIVIDADES DESARROLLADAS}

En forma conjunta con directivos y docentes de la escuela Capitán de los Andes, se acordaron los principales temas que sería conveniente abordar en las clases y también se pautaron las experiencias que se llevarían a cabo con el fin de motivar en la comunidad interés por la temática a la vez de crear una conciencia ambiental más eficiente, con la posible incorporación de energías renovables y uso racional de la energía en los lugares donde desarrolla sus actividades. La comunidad de esta escuela estaba interesada en los temas propuestos y contaba con trabajos presentados en la Feria Nacional de ciencia del año 2016.

Una vez acordados los temas de las clases y las actividades de los talleres, se desarrollaron en formato digital, cuatro clases sobre los contenidos siguientes:

\subsubsection{CONCEPTUALES}

Las diferentes fuentes de energía que existen en el mundo y en nuestro país.

Las fuentes de energia renovable y no renovable

Problemas derivados de la produccion y utilización de energía

Energías convencionales utilizadas en los edificios - uso racional y ahorro- posibles mejoras de las condiciones de habitabilidad en la casa y en la escuela.

$>\quad$ Sistemas con energia renovable, horno solar y colector solar de agua caliente sanitaria.

\subsubsection{PROCEDIMENTALES}

Diferenciación de las fuentes de energía.

Explicación de los problemas derivados de la producción y utilizacion de energía

Análisis del sol como fuente de energía en los edificios para mejorar las condiciones de habitabilidad de manera pasiva 
Análisis del sol como fuente de energía en sistemas para cocción de alimentos y para calentar agua sanitaria.

\subsubsection{ACTITUDINALES YIO EDUCACIÓN DE VALORES}

$>\quad$ Desarrollo de hábitos de consumo responsable y eficiente de energía convencional.

$>\quad$ Desarrollo de hábitos de uso de la energía solar para acondicionar naturalmente los ambientes y mejorar las condiciones de habitabilidad.

Los contenidos de las clases se plantearon de modo que resultaron accesibles para los estudiantes, incluyendo gráficos y videos con el fin de motivarlos a reflexionar sobre la temática, incorporar nuevo conocimiento y desarrollar en ellos una actitud crítica sobre los hábitos y formas de uso de la energía. Se incentivó la interacción a través del diálogo para conocer las inquietudes y reflexionar sobre los temas propuestos, propiciando la creacion colectiva del conocimiento. Se procuró vincular los conceptos con situaciones cotidianas de la casa y el aula partiendo así de un contexto que resultaba conocido y familiar para los niños.

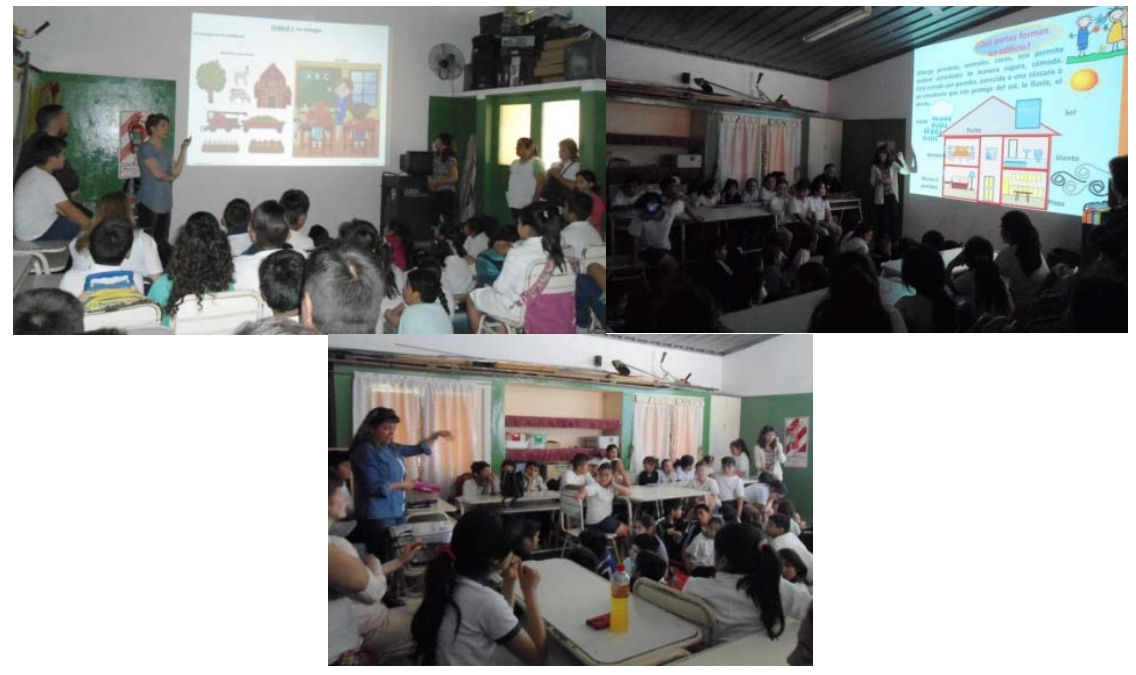

Fig. 6, 7 y 8. Presentaciones sobre Energias Renovables y No Renovables, su aplicación en edificios, uso racional y ahorro

En forma conjunta y coordinada se llevaron a cabo experiencias que incluyeron modelos demostrativos de dos sistemas con energía solar: Horno Solar y Colector de Agua. En una jornada taller con participación de toda la comunidad escolar se preparó una comida (arroz con leche) en el horno solar y se calentó agua en el colector solar. (Fig. 9 y 10). Se mostró y explicó el funcionamiento de cada uno. Luego, a través de la clase, se expusieron modelos de Horno Solar y Colector de Agua, tomando como ejemplo la utilización de diferentes materiales y accesorios, factibles de ser construidos y utilizados en la escuela y en la propia casa (Fig. 11, 12 y 13). 


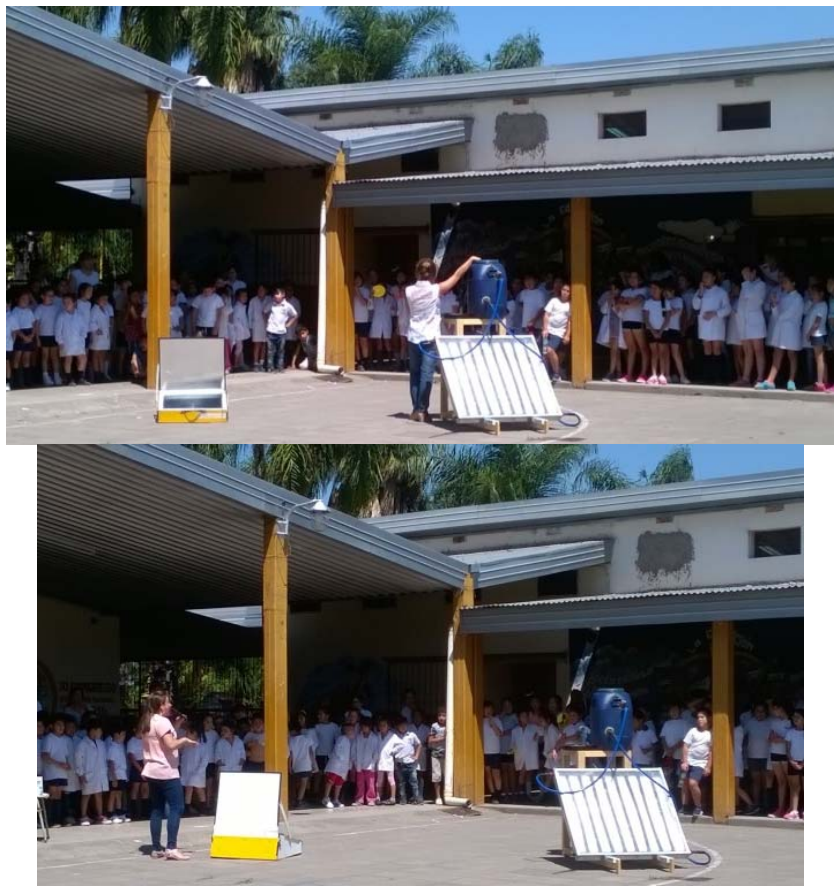

Fig. 9 y 10. Demostración de los Sistemas con Energía Renovable,Cocina Solar y Colector de Agua
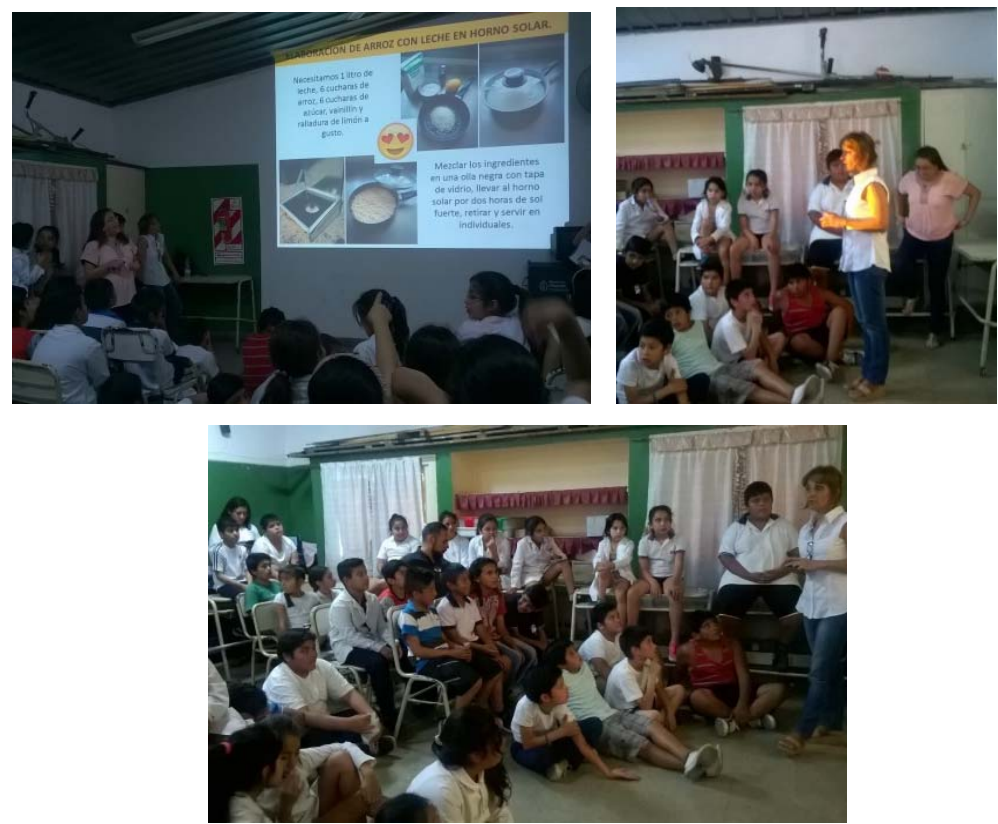

Fig.11, 12 y 13. Presentaciones sobre Sistemas con Energía Renovable,Cocina Solar y Colector de Agua.

Como actividad para la integración de los conceptos abordados, se llevó a cabo una jornada taller de actividades plásticas en la cual los estudiantes de $4^{\circ}$ y $5^{\circ}$ grado realizaron dibujos sobre los temas desarrollados en las clases (Fig. 14, 15, 16 y 17). Se consideró importante trabajar en forma interdisciplinaria y reconocer la relacion de la educación ambiental con la plástica, la cual permite enriquecer la percepción visual y al mismo tiempo imaginar la transformación de su entorno, favorecer la expresión de los sentimientos y de vivencias de los niños sobre los contenidos, relacionándolos con experiencias propias ( Fig. 18 y 19). 


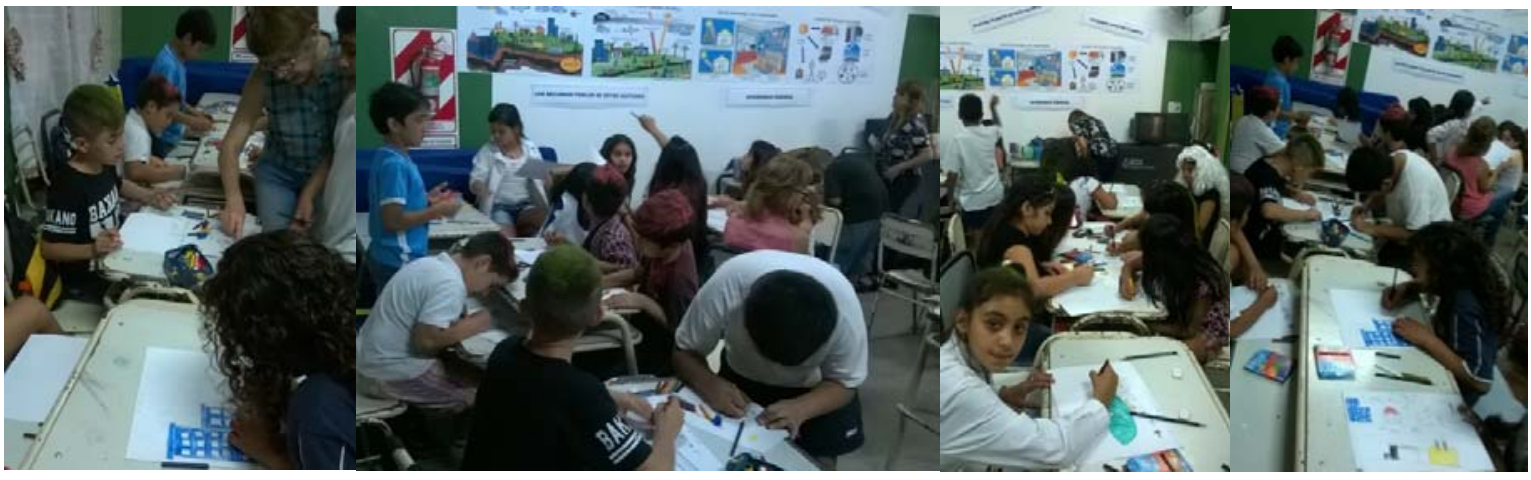

Fig. 14, 15, 16 y 17. Taller de plástica sobre los temas de las clases y sobre la experiencia con los sistemas solares

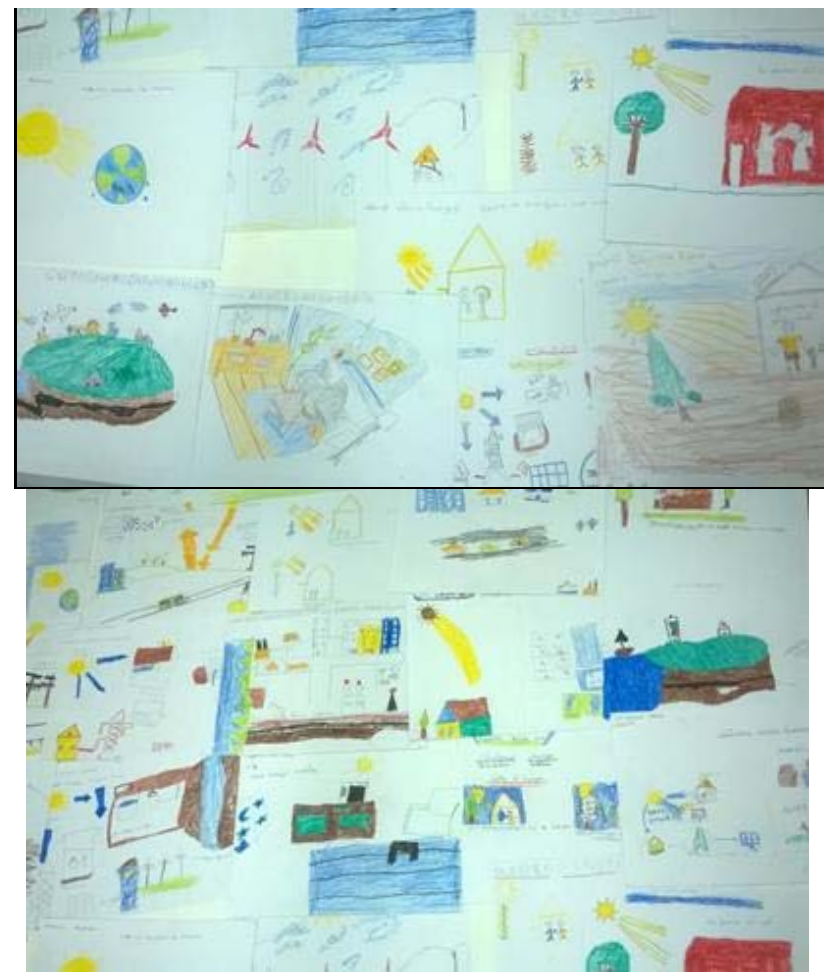

Fig. 18 y 19. Trabajos realizados por alumnos de $4^{\circ}$ y $5^{\circ}$ grado de la escuela Capitán de los Andes

Una vez finalizada la primera etapa se realizaron entrevistas a los docentes para conocer su valoración sobre la experiencia y las sugerencias de modificaciones y ajustes que consideraban necesario realizar para alcanzar los objetivos propuestos. Se les preguntó si los contenidos y la estrategia didáctica les parecía apropiada para ser aplicada en el dictado de la temática, si consideraban que era posible aplicar los contenidos abordados para desarrollar cambios en los hábitos de uso de la energía eléctrica y gas en la escuela y en la casa, y también se les planteó que realizaran sugerencias y propuestas que consideraran necesarias. La mayoría de los entrevistados manifestó una valoración positiva de la experiencia, destacando los recursos didácticos que se utilizaron, los contenidos y las estrategias de interacción con los estudiantes. Algunas de las sugerencias planteadas fueron incorporar en la experiencia a los alumnos de $3^{\circ}$ grado porque consideraban que los contenidos se adaptan a los conocimientos que los niños presentan en ese nivel y sobre la posibilidad dictar talleres dirigidos a padres y madres de 
alumnos para la autoconstruccion de los sistemas con energía solar para coccion de alimentos y para calentar agua.

En la segunda etapa se realizó la compaginacion del material utilizado en las clases y talleres con el fin de transferirlo a la escuela (Fig.20). El material consiste en un CD conteniendo cuatro clases en formato digital, sobre los temas desarrollados. Las clases incluyen también el texto explicativo que apoya la presentación de las diapositivas y los videos seleccionados. CUADERNILLOS impresos, conteniendo el desarrollo escrito y gráfico de los temas de las clases para difusión de la temática en la comunidad de la escuela. POSTERS sobre Energía y Ambiente, donde se muestran en forma gráficas y en textos sintéticos, los principales conceptos. (Fig. 21, 22 y 23).
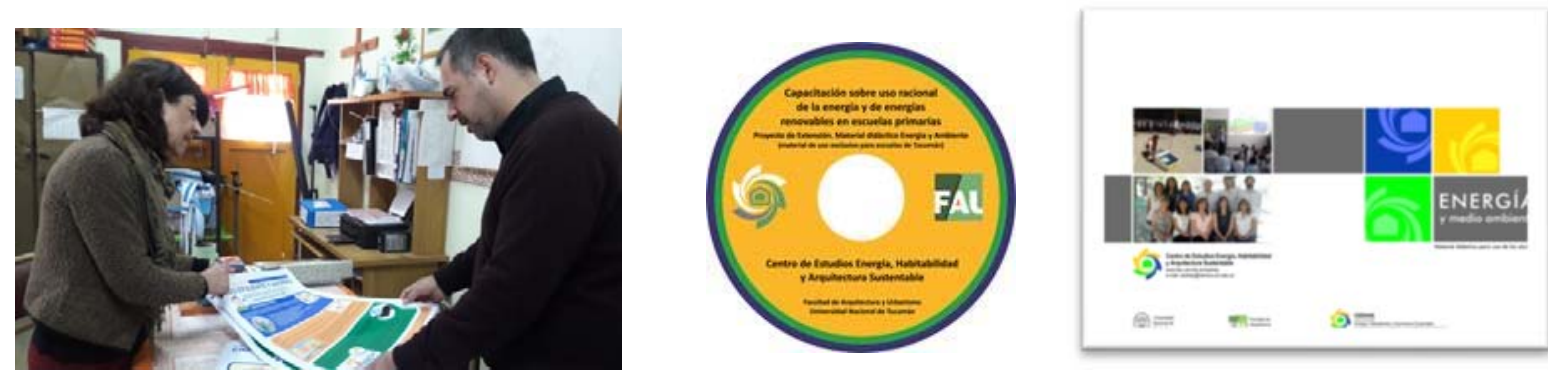

Fig. 20 Entrega de material de apoyo. Fig. 21 y 22. CD y CUADERNILLO para ser transferidos a las escuelas para difusión de la temática energético-ambiental.
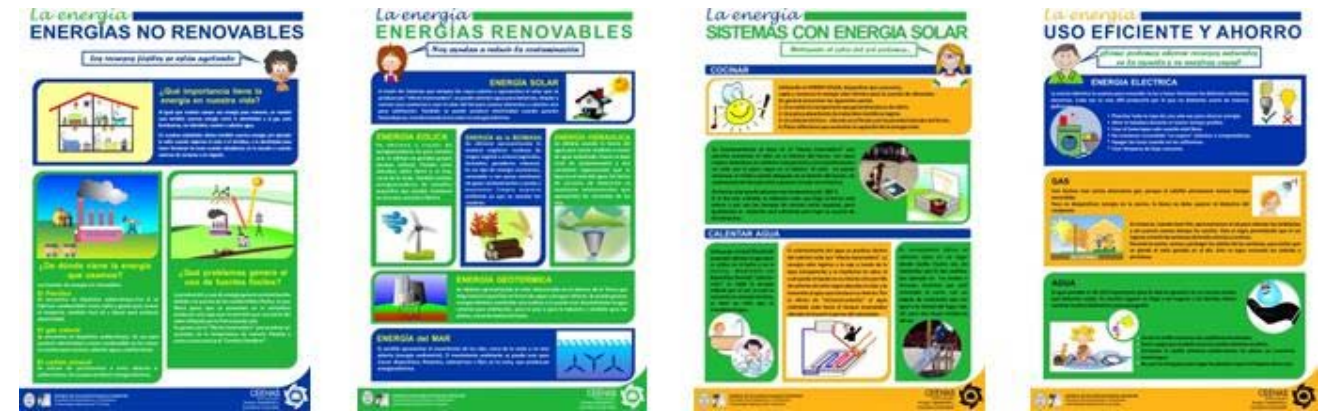

Fig. 23. PÓSTERS para ser transferidos a las escuelas para difusión de la temática energético-ambiental.

\section{CONCLUSIONES}

Las actividades desarrolladas en la escuela Capitán de Los Andes permitieron llevar a cabo los objetivos planteados en el proyecto de extensión "Capacitación y transferencia sobre mejoramiento de condiciones ambientales, uso racional de energía y energías renovables", de la cátedra de Acondicionamiento Ambiental I de la Facultad de Arquitectura y Urbanismo de la Universidad Nacional de Tucumán. El compromiso e interés manifestado por los niños en relación con los temas propuestos permitió verificar la necesidad de incorporar la temática en el nivel de educación primaria.

En base al trabajo conjunto de docentes y estudiantes, se elaboró material para transferencia y difusión sobre el tema, para ser distribuido en las escuelas: cartillas temáticas, clases y posters. También a partir de la experiencia realizada se estructuró un curso dirigido a docentes del nivel primario, el cual será implementado con la coordinación de la Secretaría de Medio Ambiente de la Provincia. 
A través de la participación de la comunidad escolar en las actividades propuestas se logró incorporar nuevos conocimientos sobre temática energético ambiental y desarrollar herramientas para la solución de problemas cotidianos. El proyecto también permitió al equipo de investigadores desarrollar técnicas y estrategias pedagógicas, el cual, si bien cuenta con una importante experiencia y conocimientos sobre el tema, se encontró ante el desafío de reelaborar conceptos para que resulten accesibles para los niños del nivel primario, al igual que el material gráfico y multimedia. Luego de la experiencia piloto, la escuela ha continuado trabajando, aplicando los contenidos en nuevas actividades y desarrollando conocimiento de forma autónoma e independiente, lo cual es valorado positivamente. La experiencia ha demostrado la importancia de trabajar en todos los niveles de la educación para colaborar en la formación de ciudadanos que reconozcan valores, aclaren conceptos y desarrollen las habilidades y las actitudes necesarias para una convivencia armónica en el contexto ambiental.

\section{BIBLIOGRAFÍA}

Blasco Lucas I. (2006). Potencial de ahorro energético en el sector residencial desde un enfoque bioclimático. FAUD-UNSJ. San Juan (Argentina)

Follari, R. (1999). La interdisciplinariedad en la educación ambiental. Tópicos en educación ambiental Vol.1, No.2 agosto. Universidad Autónoma de Aguascalientes. (México). Publicado en https://es.scribd.com/document/52603907/Interdisciplina-EA-Follari

González, E. y Figueroa, L. (2009). Los Valores Ambientales en los Procesos Educativos: Realidades y Desafíos. REICE. Revista Iberoamericana sobre Calidad, Eficacia y Cambio en Educación, 7 95-115

Moré, M. (2013): La incorporación de la educación ambiental para el desarrollo sostenible a la didáctica de las ciencias de la naturaleza, en la educación primaria de Villa Clara. (Cuba). Alternativas para su evaluación. Revista Iberoamericana de Evaluación Educativa, 6(1), 115-134.

Reyes D. (2015). Desarrollo Sustentable: Principios, objetivos, características. ITSJR Instituto Tecnológico de San Juan del Río. Orgullo Águila. (México). Categoría Cine y animaciones. Licencia estándar de YouTube. https://youtu.be/lvO5fKDLJ6A

Roque Molina, M. (2003) La Educación Ambiental: Acerca de sus Fundamentos Teóricos y Metodológicos. [en Revista Electrónica de la Agencia de Medio Ambiente] /revistame / artículo 6. $\mathrm{htm}$.

Torres, H. (2009): Experiencia de educación ambiental desde el proceso visual y el diálogo en la escuela primaria. Recuperado de http://www.monografias.com/trabajos89/experiencia-educacionambiental/

UNESCO-PNUMA Programa Internacional de Educación Ambiental, Serie Educación Ambiental 21, ACTIVIDADES DE EDUCACION AMBIENTAL PARA LAS ESCUELAS PRIMARIAS, Centro Internacional de Educación para la Conservación, Programa Internacional de Educación Ambiental (PIEA). Publicado por la Oficina Regional de Educación de la UNESCO para América Latina y el Caribe. Santiago. (Chile) julio 1997. http://unesdoc.unesco.org/images/0009/000963/096345so.pdf 